Journal of Case Reports 2017;7(3):316-318

\title{
Factor XIII Deficiency Presenting with Intra-cerebral Hemorrhage
}

\author{
Lekshmi Sudev, Nittin Henry, N.I Kurian, Susheela J Innah
}

Department of Immunohematology \& Blood Transfusion, Jubilee Mission Medical College and Research Institute, Thrissur, Kerala 680005 , India.

\section{Corresponding Author:}

Dr. Lekshmi Sudev

Email: dr.lekshmisudev@gmail.com

This is an Open Access article distributed under the terms of the Creative Commons Attribution License (creativecommons.org/ licenses/by/3.0).

Received Accepted Published
April 2, 2017

July 30,2017

September 20, 2017

\begin{abstract}
Background: Factor XIII deficiency is a rare disorder which generally presents with life threatening bleeds. Case Report: A 44 year old male presented with spontaneous left sided weakness and frontal headache for 3 days. The patient had recurrent episodes of umbilical stump bleeding and recurrent muco-cutaneous bleeds. The patient was transfused with blood components in the past. The coagulation profile showed normal prothrombin time, activated partial thromboplastin time and platelet count. Patient showed decreased clot stability with $5 \mathrm{M}$ urea solubility test. CT brain showed right temporal large hemorrhagic contusion. A parieto-occipital craniotomy and evacuation was done after transfusion of cryoprecipitate. Post-operative period was uneventful. Conclusion: The investigation by clot lysis test in the presence of a normal coagulation profile on a case with high index of suspicion is recommended. The patient was successfully managed with cryoprecipitate transfusion and supportive care.
\end{abstract}

Keywords: Blood Coagulation Tests, Brain, Craniotomy. Factor XIII Deficiency, Urea Solubility.

\section{Introduction}

Factor XIII or clot stabilizing factor is a transglutaminase enzyme which after activation crosslinks the fibrin strands thereby stabilizing the fibrin clot. It presents as a heterodimer with 2 catalytic A subunits and 2 carrier B subunits [1]. Congenital factor XIII deficiency is a rare (1 in 3-5 million people) autosomal recessively inherited severe bleeding disorder most commonly due to the deficiency of A subunit [2]. Bleeding manifestations are rare and are usually seen in $<5 \%$ of patients, usually in families with consanguinity and compound heterozygosity $[3,4]$. It is typically manifested as umbilical cord bleeding, postoperative bleeding and intracranial hemorrhage. Delayed wound healing and recurrent pregnancy loss are also observed [1]. The prime reason for bleeding is because of the inability to form a sustainable clot which tends to re-bleed after 24-48 hours. It's key role in angiogenesis affects wound healing and tissue repair.

\section{Case Report}

A forty four year old man was referred with complaints of spontaneous left sided weakness which was associated with frontal headache for the past three days. There was no history of any vomiting or loss of consciousness. The patient had recurrent episodes of bleeding in the past, most notable was a prolonged umbilical stump bleeding few days after birth. He also had recurrent mucocutaneous bleeds in the past. There is history of transfusion with multiple blood components. The child was a product of non-consanguineous marriage. There is history of sudden death of sibling at the age of 19 years. Both parents and surviving 4 siblings ( 2 sisters and 2 brothers) have no history of any coagulopathy. On examination, the patient was drowsy and disoriented. His vitals were stable and the Glasgow Coma Scale score was 10/15 (E2V3M5). Both the pupils were sluggishly reacting to light. There was loss of power of both 
his left upper and lower limbs (grade 3/5). All other system examination was within normal limits.

A computed tomography brain showed a large right temporal lobe hemorrhage. Laboratory findings showed normal hemoglobin, hematocrit and total counts. The coagulation profile showed normal prothrombin time (PT), activated partial thromboplastin time (aPTT), platelet count and bleeding time. In view of the normal coagulation parameters and the history of prolonged umbilical stump bleeding at birth, a differential diagnosis of factor XIII deficiency was considered. 5M urea solubility clot lysis test showed decreased clot stability. Factor XIII deficiency with intra-cerebral hemorrhage was the final diagnosis.

Patient was transfused with cryoprecipitate and was taken up for surgery. A parieto-occipital craniotomy and evacuation was done. Seven units of cryoprecipitate were given prior to the surgery. This was followed by a repeat dose of seven units two days after the surgery. Post-operative period was uneventful and the patient was discharged one week after the surgery. The patient was advised to be on prophylactic FXIII replacement either with fresh frozen plasma or with cryoprecipitate at the Comprehensive Hemophilia Treatment center.

\section{Discussion}

The presence of multiple mucocutaneous bleeds, profuse umbilical stump bleeding following birth and presently the development of intracerebral hemorrhage in the presence of a normal coagulation profile directed us towards performing $5 \mathrm{M}$ urea solubility test. The incidence of umbilical stump hemorrhage and intra-cerebral hemorrhage in factor XIII deficiency is reported to be $80 \%$ and $25-30 \%$ respectively [4]. The investigation by $5 \mathrm{M}$ urea solubility test in the presence of a normal coagulation profile (PT, APTT, bleeding time and platelet count) in a case with high index of suspicion is highly recommended. A 5M urea solubility test positive for lysis is only seen with severe deficiency of factor XIII (1-3\%) [4]. Limitations in our case study were not doing factor XIII assay and failure to provide factor XIII concentrates due to financial constraints. The normal level of factorXIII activity is 50-220\% [2]. Ideally the treatment for acute bleeding should be decided based on thromboelastography (TEG), clot solubility, history of bleeding and the severity of bleed. Factor XIII levels should be maintained at $10-20 \%$ to prevent further bleeding and also poor wound healing. There are not many controlled trials to validate the dosage in a similar acute setting. Transfusion of fresh frozen plasma or cryoprecipitate transfusion itself is a highly effective treatment. This is because only small quantities of factor XIII (approximately $5-30 \%$ ) are needed for maintaining coagulation and factor XIII has a comparatively longer halflife of 9-14 days. Plasma derived virus inactivated factor XIII is available but is being slowly replaced by recombinant factor XIII A2 which is the present treatment of choice. Patients with intracranial hemorrhages that occurs secondary to factor XIII deficiency should be prophylactically supplemented with factor XIII supplementation either as fresh frozen plasma (FFP $10 \mathrm{ml} / \mathrm{kg}$ every 4-6 weeks), cryoprecipitate transfusion (1 unit cryoprecipitate for $10-20 \mathrm{~kg}$ every 3-4 weeks) or factor XIII concentrates (10-35 U/kg every 4-6 weeks) [4-7]. The patient was successfully managed with cryoprecipitate transfusion and supportive care.

\section{Conclusion}

A high index of suspicion based on medical history, family history followed by $5 \mathrm{M}$ urea solubility test is instrumental in diagnosing congenital FXIII deficiency, though a patient presenting undiagnosed so late with respect to his age is quite rare. Prophylactic factor XIII supplementation is highly recommended to prevent similar episodes in the future. 
Contributors: LS: manuscript writing, literature search; NH: manuscript editing, literature search; NIK, SJI: Study designing and manuscript editing. LS will act as guarantor. All authors approved the final version of manuscript.

Funding: None; Competing interests: None stated.

\section{References}

1. Kaushansky K, Marshall AL, Beutler E, Kipps TJ, Seligshon U, Parchal JT. Williams Hematology; $8^{\text {th }}$ edition. Newyork, NY:Mc Graw Hill Publishers;2011:2042-2045.

2. Peyvandi F, Duga S, Akhavan S, Mannucci PM. Rare coagulation deficiencies: Haemophilia. 2002;8:308-321.
3. Hsieh L, Nugent D. Factor XIII deficiency. Haemophilia. 2008;14:1190-1200.

4. Anwar R, Miloszewski KJ. Factor XIII deficiency. Br J Haematol. 1999;107:468-484.

5. Robberts HR, Bingham MD. Other coagulation factor deficiencies. In: Loscalzo J, Schafer AI, eds. Thrombosis and Haemorrhage, $3^{\text {rd }}$ edn. Philadelphia, PA: Lippincott Williams and Wilkins; 2003:592-593.

6. Inbal A, Oldenburg J, Carcao M, Rosholm A, Tehranchi R, Nugent D. Recombinant factor XIII: a safe and novel treatment for congenital factor XIII deficiency; Blood. 2012;119:5111-5117.

7. Nugent D. Prophylaxis in rare coagulation disorder: factor XIII deficiency. Thromb Res. 2006;118:523-528. 